Revue

Revue de l'histoire des religions

de Ihistoire des religions

3 | 2012

Varia

Ricardo ESCOBAR QUEVEDO, Inquisición y judaizantes en América española (siglos XVI-XVII)

[prólogo de Charles AMIEL], Bogotá, Editorial Universidad del Rosario («Textos de Ciencias Humanas »), 2008, 438 p., 24 cm, ISBN 978-958-837856-5.

Bruno Feitler

\title{
OpenEdition
}

Édition électronique

URL : http://journals.openedition.org/rhr/7951

DOI : 10.4000/rhr.7951

ISSN : 2105-2573

Éditeur

Armand Colin

Édition imprimée

Date de publication : 1 septembre 2012

Pagination : 449-452

ISBN : 978-2200-92975-0

ISSN : 0035-1423

Référence électronique

Bruno Feitler, «Ricardo escobar quevedo, Inquisición y judaizantes en América española (siglos XVI-XVII) », Revue de l'histoire des religions [En ligne], 3 | 2012, mis en ligne le 04 octobre 2012, consulté le 22 septembre 2020. URL : http://journals.openedition.org/rhr/7951 ; DOI : https://doi.org/10.4000/rhr. 7951

Ce document a été généré automatiquement le 22 septembre 2020

Tous droits réservés 


\section{Ricardo ESCOBAR QUEVEDO, Inquisición y judaizantes en América española (siglos XVI-XVII)}

[prólogo de Charles AMIEL], Bogotá, Editorial Universidad del Rosario (« Textos de Ciencias Humanas »), 2008, 438 p., $24 \mathrm{~cm}$, ISBN 978-958-837856-5.

\section{Bruno Feitler}

\section{RÉFÉRENCE}

Ricardo ESCOBAR QUEVEDO, Inquisición y judaizantes en América española (siglos XVI-XVII), [prólogo de Charles AMIEL], Bogotá, Editorial Universidad del Rosario (« Textos de Ciencias Humanas »), 2008, 438 p., 24 cm, ISBN 978-958-837856-5.

1 Le phénomène des judaïsants, ainsi que celui des conversos en général, ne peut être bien appréhendé sans la compréhension de l'histoire de l'Inquisition. Le livre de Ricardo Escobar, issu de sa thèse de doctorat, soutenue à l'École des Hautes Études en Sciences Sociales en 2003 sous la direction de Charles Amiel, le démontre parfaitement. En effet, comme l'auteur l'affirme à la fin de son introduction, en Amérique (mais c'est également vrai pour l'Europe), «le destin des judaïsants et des inquisiteurs est curieusement imbriqué : antagonistes liés à une dialectique qui ne cesse de les opposer, mais qui, quelquefois, les rapproche ». Il rappelle aussi l'ironie du fait qu'on doit justement à cette institution qui se vouait à l'anéantissement de l'hérésie judaïsante, la perpétuation, grâce à ses archives, de son histoire. Cette étude essaye donc de retracer l'histoire des groupements judaïsants de l'Amérique espagnole en la liant aux rythmes de persécution. Au-delà, elle rend compte non seulement de la complexe histoire religieuse de ceux qu'on appelle aussi les "marranes", mais également de leur importance économique pour les échanges atlantiques. Elle fournit ainsi une importante contribution empirique à l'histoire des réseaux, montrant les flux de 
communication existant entre Manille, Mexico, Lima, Carthagène des Indes et Séville ou Lisbonne, mais aussi ceux, moins évidents, qui liaient les conversos ou nouveauxchrétiens des centres américains au sud de la France, à l'Italie, aux Pays-Bas et aux ports négriers de l'Afrique de l'Ouest. Ainsi donc la principale contribution de cette étude réside dans la perspective globale à partir de laquelle l'auteur présente la constitution des groupes conversos dans l'Amérique espagnole.

2 L'ouvrage est organisé autour de cinq parties intitulées: "Diaspora et cryptojudaïsme », "Le Saint-Office aux Indes ", "L'offensive inquisitoriale », "Les nouveaux-chrétiens et le commerce des esclaves » et «Les pratiques religieuses ». Les trois premières retracent l'histoire de quelques familles de nouveaux-chrétiens originaires du bourg de Castelo Branco au Portugal qui donnèrent naissance à une bonne partie des personnes arrêtées par l'Inquisition pour judaïsme en Amérique dans les années 1630-40. Passées d'abord en Espagne, en plusieurs vagues migratoires provoquées par la persécution inquisitoriale au Portugal, surtout entre 1580 et les années 1610, certaines familles s'installent à Séville et poursuivent leur odyssée en direction du nouveau monde, sans que se perde la mémoire de l'origine portugaise et du centre sévillan. Ce récit est entrecoupé par de larges parenthèses contenant des mises au point sur la question marrane ou rendant compte de l'histoire et des procédures inquisitoriales. La quatrième partie affine l'histoire de ces groupes autour de la question de la traite des esclaves, et la dernière passe au crible les rites, les bases doctrinales et les complexes origines des pratiques cryptojuives.

Dans l'ensemble, se dégage donc l'histoire de ces familles dans une perspective globale qui finit par dépasser la description de leurs aléas spécifiques pour rendre compte de modèles plus significatifs. En étudiant les 617 cas de judaïsants enregistrés dans les trois tribunaux inquisitoriaux américains entre la fondation des Inquisitions de Mexico et de Lima en 1569 et l'année 1669, lorsque le nombre de cas de judaïsme décline définitivement, Ricardo Escobar montre d'abord l'origine commune des prévenus située dans ce village montagneux de la frontière espagnole, ce qui indique l'importance non seulement des racines juives, mais aussi géographiques et familiales pour le maintien des contacts et la formation de réseaux. Ensuite, il apparaît que cette origine commune donne toutefois lieu à deux genres de comportements sociologiques et religieux différents, qui peuvent en gros être expliqués par des choix économiques. Tandis qu'en Nouvelle Espagne nous trouvons un modèle de migration définitive de couples avec enfants et collatéraux, au Pérou et en Nouvelle Grenade ce sont surtout des hommes seuls, liés au trafic négrier (jusqu'à en devenir les maîtres), installés localement de façon provisoire, qui sont arrêtés par l'Inquisition. Cette typologie - qui sans doute met les territoires du Sud, ceux où se sont installés ces judaïsants, dans la condition de territoires de frontière -, sert aussi à décrire les différents modes de pratique du cryptojudaïsme américain. En Nouvelle Espagne dominait une vigoureuse transmission familiale des coutumes et des rites judaïsants, tandis qu'au Pérou et en Nouvelle Grenade les croyances et pratiques juives semblent beaucoup plus faibles, même si d'importantes exceptions existent, avec des cas bien connus de martyre, qui résultent, selon l'auteur, de leur isolement par rapport au courant principal judaïsant (p. 81-82).

4 C'est justement la mise en perspective historique du cryptojudaïsme respecté par ces familles qui est un des points forts du livre. Traditionnellement, l'historiographie depuis Cecil Roth a vu le phénomène marrane comme quelque chose d'éternel qui, sous 
le couvert du secret, transmettait de génération en génération la connaissance du judaïsme originel. Si depuis lors on a reconnu l'inexorable affaiblissement des croyances et des rites cryptojuifs dû justement à leur caractère secret, Ricardo Escobar montre ici qu'il n'en est rien, mais qu'il y a eu, pour ces groupes étudiés, un « processus de consolidation doctrinale", des moments dans lesquels "les pratiques religieuses sont actualisées et homogénéisées» (p.319), par des "dogmatistes» ayant fait l'expérience des communautés juives normatives d'Italie et de l'Europe du Nord ou semi-clandestines du sud de la France. Il faut aussi attirer l'attention sur le rôle, déjà plus connu, de l'écrit dans la transmission des connaissances juives (p. 289-292).

5 Également importante à noter est l'ouverture sociale, limitée car dictée par la norme de l'endogamie, de ces groupes de judaïsants. En raison de leur importance dans le trafic négrier, ils intégrèrent des femmes africaines et ensuite des métisses, qui eurent un rôle non négligeable dans la transmission du cryptojudaïsme (p. 62-64). Cette constatation montre encore une fois que, pour bien comprendre l'histoire des nouveaux-chrétiens et des judaïsants, il faut abandonner les catégories traditionnelles, et aussi contemporaines, de la transmission familiale close et exclusiviste du judaïsme. Pour donner un autre exemple, dans l'Amérique portugaise cette fois, des études montrent que sur les quelque 300 nouveaux-chrétiens arrêtés pour judaïsme à Rio de Janeiro dans la première moitié du XVIII ${ }^{e}$ siècle, environ 50 , soit $16,7 \%$, avaient des origines africaines et/ou amérindiennes ( $c f$. Lina Gorenstein Ferreira da Silva, A Inquisição contra as mulheres. Rio de Janeiro, séculos XVII e XVIII, São Paulo : Humanitas, 2005, p. 268-271).

6 Finalement, pour relier à nouveau la question de la persécution avec l'histoire spécifique des tribunaux inquisitoriaux, Ricardo Escobar essaye de montrer qu'il convient de dépasser la lecture traditionnelle des archives inquisitoriales qu'ont les historiens du marranisme. Une bonne connaissance du fonctionnement même de l'institution ainsi que du comportement de ses ministres permet de bien cerner la destinée de ceux qui passaient par les cachots du Saint-Office. Il analyse ainsi la promiscuité existant entre nouveaux-chrétiens et inquisiteurs, tant au Mexique qu'à Carthagène, et le rôle que la personnalité d'un inquisiteur pouvait jouer dans le jugement qu'il rendait : à preuve d'exemple le cas de Juan Sáenz de Mañozca, membre d'un véritable clan inquisitorial, d'où sortirent également un oncle secrétaire et un cousin inquisiteur. L'action des deux cousins dans les tribunaux de Lima et de Mexico est essentielle pour une réelle compréhension des différences avérées dans les types de peines infligées selon le lieu et le moment.

7 L'ouvrage, dans un souci de clarté, comporte plusieurs généalogies, tant dans le corps du texte que dans les annexes. Il inclut également des tableaux statistiques sur la persécution des judaïsants ainsi que la transcription de documents sur les accusations à l'encontre des inquisiteurs du tribunal de Carthagène lors des visites effectuées au nom du Conseil suprême de l'Inquisition (la Suprema) en 1643 et en 1650. 


\section{AUTEURS}

\section{BRUNO FEITLER}

Universidade Federal de São Paulo. 